\title{
Analyzing the proximity to cover in a landscape of fear: A new approach applied to fine-scale habitat use by rabbits facing feral cat predation on Kerguelen archipelago
}

Pierrick Blanchard, Christine Lauzeral, Simon Chamaillé-Jammes, Nigel G Yoccoz, Dominique Pontier

Although proximity to cover has been routinely considered as an explanatory variable in studies investigating prey behavioral adjustments to predation pressure, the way it shapes risk perception still remains equivocal. This paradox arises from both the ambivalent nature of cover as potentially both obstructive and protective, making its impact on risk perception complex and context-dependent, and from the choice of the proxy used to measure proximity to cover in the field, which leads to an incomplete picture of the landscape of fear experienced by the prey. Here, we study a simple predator-prey-habitat system, i.e., rabbits Oryctolagus cuniculus facing feral cat Felis catus predation on Kerguelen archipelago. We assess how cover shapes risk perception in prey and develop an easily implementable field method to improve the estimation of proximity to cover. In contrast to protocols considering the "distance to nearest cover", we focus on the overall "area to cover". We show that fine-scale habitat use by rabbits is clearly related to our measure, in accordance with our hypothesis of higher risk in patches with smaller area to cover in this predator-prey-habitat system. In contrast, classical measures of proximity to cover are not retained in the best predictive models of habitat use. The use of this new approach, together with a more in-depth consideration of contrasting properties of cover, could help to better understand the role of this complex yet decisive parameter for predator-prey ecology. 


\section{Analyzing the proximity to cover in a landscape of fear:}

2 A new approach applied to fine-scale habitat use by rabbits facing feral

5 Pierrick Blanchard ${ }^{1}$, Christine Lauzeral ${ }^{1}$, Simon Chamaillé-Jammes ${ }^{2}$, Nigel G. Yoccoz ${ }^{3}$ and

6 Dominique Pontier ${ }^{4}$

7

8

9 'Université de Toulouse, Université Toulouse III Paul Sabatier, CNRS, ENFA, UMR 5174 (Laboratoire Évolution et

Diversité Biologique), F-31062 Toulouse, France

${ }^{2}$ Centre d'Écologie Fonctionnelle et Évolutive, CNRS, UMR 5175, F-34293 Montpellier Cedex 5, France

${ }^{3}$ Department of Arctic and Marine Biology, UiT The Arctic University of Norway, NO-9037 Tromsø, Norway

${ }^{4}$ Université de Lyon, Université Lyon I Claude Bernard, CNRS, UMR 5558 LBBE (Laboratoire Biométrie et

Corresponding author :

17 Pierrick Blanchard

18 Université Paul Sabatier, 118, route de Narbonne, 31062 TOULOUSE Cedex 09, France 
20 Abstract

21 Although proximity to cover has been routinely considered as an explanatory variable in studies

22 investigating prey behavioral adjustments to predation pressure, the way it shapes risk perception

23 still remains equivocal. This paradox arises from both the ambivalent nature of cover as

24 potentially both obstructive and protective, making its impact on risk perception complex and

25 context-dependent, and from the choice of the proxy used to measure proximity to cover in the

26 field, which leads to an incomplete picture of the landscape of fear experienced by the prey.

27 Here, we study a simple predator-prey-habitat system, i.e., rabbits Oryctolagus cuniculus facing

28 feral cat Felis catus predation on Kerguelen archipelago. We assess how cover shapes risk

29 perception in prey and develop an easily implementable field method to improve the estimation

30 of proximity to cover. In contrast to protocols considering the "distance to nearest cover", we

31 focus on the overall "area to cover". We show that fine-scale habitat use by rabbits is clearly

32 related to our measure, in accordance with our hypothesis of higher risk in patches with smaller

33 area to cover in this predator-prey-habitat system. In contrast, classical measures of proximity to

34 cover are not retained in the best predictive models of habitat use. The use of this new approach,

35 together with a more in-depth consideration of contrasting properties of cover, could help to

36 better understand the role of this complex yet decisive parameter for predator-prey ecology. 


\section{Introduction}

Cover, hereafter defined as any tangible feature in the habitat that impairs the prey's and/or the predator's ability to see and/or move [i.e., the definition of "structural cover" by Mysterud and Østbye (1999), restricted to the "landscape of fear" context of Laundré et al. (2010)], has often been considered as an explanatory variable in field studies investigating prey behavioral adjustments to predation risk (Caro 2005). Yet, the way cover shapes risk perception in prey species and their subsequent antipredator tactics still remains equivocal (e.g., Burger et al. 2000, Tchabovsky et al. 2001, Caro 2005). This may be due to the ambiguity inherent to the use of a single word to refer to multiscale habitat objects (e.g., a prey may have its visual field impaired by a tree line hundreds of meters away and by a tuft of vegetation near its eyes when feeding head down) or to the paucity of studies considering simultaneously these different scales (e.g., Pays et al. 2012). Two additional reasons may explain why the role of this parameter remains ambivalent.

First, cover is a (visual/physical) barrier for the focal individual prey but also for its predator(s). Hence, the ratio between its contrasting obstructive (i.e., prevents the prey from seeing or escaping the predator) and protective (i.e., prevents the predator from seeing or attacking the prey) properties (Lazarus and Symonds 1992, Mysterud and Østbye 1999) and thus the overall risk perception is highly specific to a predator(s)-prey system. This obstructive/protective ratio depends on the intrinsic physical properties of the cover itself (e.g., dimensions, opacity), in relation to characteristics such as the body size, sensory capabilities, and hunting/escape tactics of particular predator and prey species (e.g., Lima 1990, Murray et al. 1995, Newberry and Shackleton 1997). Furthermore, intraspecific variability is expected in the 
60 above described characteristics and also in other traits (e.g., sex, reproductive status, experience

61 or group size) that may also determine risk perception in relation to a specific cover type (e.g.,

62 Götmark and Hohlfält 1995, Bowyer et al. 1999, Stratmann and Taborsky 2014, Beauchamp

63 2014). Finally, time of day (e.g., Moreno et al. 1996) or season (e.g., Bowyer et al. 1999) may

64 affect the obstructive/protective ratio for a given cover type and individual. Hence, the

65 contrasting results of studies investigating the role of cover in shaping risk perception, even

66 those performed on the same species and type of cover (e.g., Jaksic and Soriguer 1981, Moreno

67 et al. 1996), may reflect natural heterogeneity.

The second reason why the role of cover for prey remains ambivalent is that field

69

and predator-prey system, the obstructive/protective ratio of cover may vary with proximity. For instance, cover provided by drooping branches has a low obstructive/protective ratio when a prey is close because the prey is less visible but its ability to detect a predator and flee is unaffected. As distance from the branches increases, the ratio increases because the prey becomes more visible and the branches provide a possible ambush site for a predator. Hence, field studies classically consider the "distance to cover" (when mentioned, typically the "nearest" "principal" cover) as a routine measurement (Caro 2005). We speculate that part of the variability in the results of studies relating prey behavioral traits to cover is a consequence of using a single distance measurement that leads to an incomplete picture of the landscape of fear experienced by the prey. The problem with distance to nearest cover is that this measure ignores the dimension of the edge of the cover the prey faces and the presence of additional cover. Yet, in a case of an overall obstructive cover, a forest edge of 100 meters long provides more potential ambush sites than a patch of trees of 10 meters long even if the shortest distance between the cover and the 
83 prey is the same in both situations. Similarly, a prey surrounded by several shrubs is expected to

84 be at higher risk than if there is a single shrub in its vicinity in case of obstructive cover. Hence,

85 although the shortest distance to cover is of importance as this gives an indication of the shortest

86 time lag before being predated (or sheltered in case of overall protective cover), it is only part of

87 the information. There is thus a need for a measure of all distances between the prey position and

88 cover in the surroundings (see also Metcalfe 1984 and Gómez-Serrano and López-López 2014),

89 i.e., a need for an area to cover. We suggest that such a metric would provide a more reliable

90 measure of the proximity to cover and thus, of risk perception, than the commonly used

91 "distance to the nearest cover". In the present paper, our aims were to (1) develop such a metric,

92 from field measurements to their geometrical analysis and to (2) use this metric to investigate

93 habitat use of rabbits (Oryctolagus cuniculus) facing predation threat by feral cats (Felis catus)

94 on the Kerguelen subantarctic archipelago. We also considered classical proxies of proximity to

95 cover in order to allow comparisons.

96 In studies of responses to cover, there is a risk of circularity if the property of cover is

97 inferred from its effect on behavior (Lazarus and Symonds 1992). Instead, cover property, and

98 thus the way it is expected to trigger risk perception in prey, should arise from the knowledge of

99 the system. Hence, making a prediction requires assessing the risk perception (i.e., the

100 obstructive/protective ratio) inherent to the (different types of) cover(s), i.e., being able to relate

101 the physical characteristics of the cover such as opacity and size to the escape tactics of the prey

102 and the hunting tactics of the predator involved. Furthermore, it is important to recognize that

103 cover may be associated with foraging profitability (e.g., Morgantini and Hudson 1985), for

104 example if it directly provides food or affects food plants through shading or nutrients (e.g.,

105 Mysterud and Østbye 1999, Dellafiore et al. 2014). In the present paper, we took advantage of a 
106 simple predator-prey-habitat system allowing us to hypothesize that predation threat was the

107 main driving force of habitat use by rabbits. Given the specific characteristics of the system, we

108 predicted that rabbits should avoid patches with high proximity to cover, i.e., with small area to 109 cover.

111 Materials and Methods

113 Study site

114 Introduced by sailors during the nineteenth century, rabbits are now widespread

115 throughout the Kerguelen archipelago (Chapuis et al. 1994). Domestic cats were introduced in

1161951 to control invasive rodents (Rattus rattus, Mus musculus) and rabbits at the research station

117 of Port-aux-Français. Cats are now widely distributed over the main island (Grande Terre),

118 where the study took place (Pointe Morne area, 49²2’S, 70²6’E).

119 Our study was performed in December 2014. We focused on a ca. 100 by $700 \mathrm{~m}$ area

120 covered by mounds less than $2 \mathrm{~m}$ high, formed of earth and roots and covered by the perennial

121 herb Acaena magellanica (Rosaceae) (see Supplemental information file 1). The space between

122 the mounds was composed of Acaena magellanica, Poa annua and bare ground/rocks. The study

123 area is surrounded by open meadows with flat topography, covered with dense swards of Acaena

124 magellanica.

125 We censused active rabbit burrows as indicated by fresh pellets or evidence of animal 126 passage in a $0.73 \mathrm{~km}^{2}$ area including the patches (see below) used in this study. We found 51

127 active burrows, all outside our $70,000 \mathrm{~m}^{2}$ study area. Although we do not know the relationship

128 between the number of active burrows and the population size in this habitat, previous published 
129 relationships suggest about 22 rabbits (i.e., about 30 rabbits $/ \mathrm{km}^{2}$ ) (Ballinger and Morgan 2002).

130 Fifteen additional active burrows were present outside the $0.73 \mathrm{~km}^{2}$ area, $800 \mathrm{~m}$ away from the 131 study area.

132

Predicting the effect of area to cover on habitat use by rabbits

The following characteristics of our system allowed us to reliably assess the role of cover

135

136

137

138

139

140

141

142

143 in shaping risk perception in rabbits.

— Food resources. We selected patches of a single preferred plant species, Poa annua (Chapuis et al. 1994). This highly nutritious alien grass represents most of rabbit diet in our study area (over $90 \%$ of the plant fragments found in fecal pellets at the time of the year our study took place, Boussès et al. 1988). As the study area is relatively restricted, meteorological and edaphic conditions are very similar. Moreover, Poа апnua was heavily grazed (1 - $2 \mathrm{~cm}$ high) throughout the study area. Because patches were likely to be similar in food quality and quantity, the effect of cover was not confounded by foraging profitability. Finally, rabbits face no interspecific competition for feeding resources in this habitat (in particular, reindeer Rangifer tarandus have not been observed in the study area).

- Predators. Predation by brown skua (Catharacta lonnbergi) on rabbits occurs on the Kerguelen archipelago, but mostly on small islands (Chapuis et al. 1994) and on young/sick rabbits (the myxomatosis virus was introduced in 1950's to control populations). Given that our study site was on the main island, that no skuas nested nearby, that no rabbits were observed or killed (as part of other protocols) with apparent signs of myxomatosis, that our study took place before the rabbit birth period, that cats were observed daily in our study area and finally that rabbits are the primary prey of cats in Kerguelen archipelago (Pontier et al. 2002), we believe 
152 that predation pressure experienced by rabbits in this habitat is mostly due to cats. This contrasts

153 with other studies on rabbits and other prey where predators are often diverse. Our field

154 observations of hunting bouts revealed that cats are stalk-and-ambush predators (although they

155 also visit burrows). A foraging rabbit is clearly at risk if surprised by a cat, while early visual

156 detection of the cat allows escape, especially in open areas.

157 - Cover types. We focused on a habitat with a single type of cover, earth mounds (i.e.,

158 visually opaque and physically impenetrable, see Supplemental information file 1), and

159 considered as "cover object" any mound higher than $20 \mathrm{~cm}$ (i.e., capable of hiding an ambushing

160 cat from a rabbit, even in an upright posture, and of hiding a rabbit from a cat, unless the rabbit

161 was in an upright posture). Most mounds were taller than $1 \mathrm{~m}$. Cats may attack directly from a

162 side of a mound, but also from behind a smaller mound or possibly from the top of a larger

163 mound.

164 The characteristics of this predator-prey-habitat system allowed us to consider cover as a 165 source of risk for rabbits, i.e., far more obstructive (total opacity in a context of stalk-and-

166 ambush predator threat and complete physical barrier when escaping, with no intrinsic refuge

167 property - once potential burrows (see below) are statistically accounted for) than protective

168 (rabbits hidden from cats). Accordingly, we predicted that rabbits would favor patches with

169 larger areas to cover, i.e., with larger unobstructed areas.

170

"Patch" characterization and data collection

172

We defined a "patch" as a circular area with a 2 m diameter, covered exclusively with

173 Poa annua, whose center was at least $20 \mathrm{~m}$ away from the center of another patch. The studied

174 area was fully searched for patches, which numbered 32. 
We used total dry weight of pellets per patch as a measure of patch use. In every patch,

176 we made a one-time collection of all the fecal pellets, thereby assuming that the disappearance

177 time of pellets was not related to the variables we considered. We also kept the pellet collection

178 time very short (2 days) to reduce the occurrence of additional pellets during the study period.

179 Pellets were subsequently dried for 4 days at $40^{\circ} \mathrm{C}$ (i.e., until their weight stopped decreasing)

180 and weighed. Pellet quantity is a reliable method to assess rabbit abundance at the habitat scale

181 (Palomares and Delibes 1997, Palomares 2001, Cabrera-Rodriguez 2006). At a finer scale, pellet

182 quantity has been shown to index the frequency of rabbit visitation of the patches (Bakker et al.

183 2005).

In every patch, a single observer took the following measurements:

— The GPS coordinates. This allowed us subsequently to investigate statistically the spatial structure of our response variable, the pellet total dry weight.

— The total number of old burrows within a $20 \mathrm{~m}$ of diameter circle around the center of the patch. This parameter was recorded because we observed that old burrows represented potential refuges. We considered the hypothesis that there were more rabbits closer to active burrows (all localized outside our study area, see above) as part of our investigation of spatial structure. The number of burrows is classically part of rabbit habitat selection studies (e.g., Palomares and Delibes 1997).

- The terrestrial distance $(\mathrm{m})$ to the nearest old burrow, bypassing a mound if present. — The number of "contact points" with Poa annua around the focal patch. Contact points were located 1, 3 and $5 \mathrm{~m}$ from the center of the focal patch every $45^{\circ}$ (i.e., $\mathrm{n}=24$ in total for each patch). A proxy of the isolation of the focal patch of Poa annua was then calculated as the frequency of "contact points" without Poa anпua. We included this parameter because we 
198 hypothesized that the attraction of a patch could have been positively related to how isolated it

199 was from other patches of Poa annua or conversely, that patches surrounded by a high overall

200 Poa annua/Acaena ratio could have been more attractive for rabbits. Moreover, this measure

201 allowed us to investigate the hypothesis that risk perception would be increased if abundant

202 Acaena around the focal patch impaired the visual field of a rabbit feeding head down.

203 - The distances $(\mathrm{m})$ from the center of the patch to the nearest mound and to the nearest

204 side of a mound visible from the patch, within a biologically relevant area (see below). In

205 addition to their biological relevance as an obstacle when escaping or a point from which a

206 hidden cat may attack, these distances allow us to compare our proposed measure with other

207 measures of risk.

208 - The total number of mound sides visible from the patch inside the biologically

209 relevant area (see below) and the mean distance (m) to these sides.

210 - Additionally, we recorded the unobstructed area for each patch. The description of this

211 measure is presented below.

212

213 Measuring the unobstructed area

214 From the center of the patch, the observer scanned $360^{\circ}$ using a rangefinder with angle

215 display (Vector 1500 GMD). Each time the rectilinear edge of a mound (i.e., forming a straight

216 line as a whole) started and stopped, the distance from the center of the patch and the

217 corresponding angle were recorded. When a mound edge did not appear to be rectilinear, the

218 measurements were recorded for each of its rectilinear segments. This gave us a set of triangles

219 with an angle and the length of the two adjacent sides, allowing the calculation of their area.

220 However, the sum of these areas would provide a poor proxy of the unobstructed area 
221 experienced by a rabbit because some mounds would be too far away to provide a cat with a

222 successful ambush position or to provide a physical barrier to escape. Furthermore, considering

223 such distant mounds may inflate the unobstructed area, potentially masking biologically relevant

224 effects at shorter distances. We thus calculated the unobstructed area within a circle. Considering

225 such a circle also allowed us to deal with cases where no cover occurred before the horizon (a

226 single sector in a single patch for our study area). We set the circle radius based on our field

227 observations of hunting behavior by cats. Because the longest cat run toward a feeding rabbit that

228 we observed was about $25 \mathrm{~m}$, we first considered this distance. Then, in order to identify the

229 radius of the circle with the highest predictive power, we computed the squared coefficient of

230 determination between the observed and the fitted values for models built with values of

231 unobstructed areas calculated for circles with radiuses ranging from 1 to $150 \mathrm{~m}$ (with $1 \mathrm{~m}$

232 increments).

233 Depending on whether the rectilinear edge of the mound the observer faced fell entirely

234 inside the circle, entirely outside the circle or was secant in one or two points, we used different

235 formulas to calculate the corresponding area, as explained Fig. 1A, B and C (see also the script

236 used to compute the unobstructed area, written in the R language and provided in Supplemental

237 information file 2). The sum of these areas provides the unobstructed areas, ranging from 25.3 to

$2381646.1 \mathrm{~m}^{2}$ for the circle with a radius of $25 \mathrm{~m}$.

239

\section{Statistical analyses}

241 The pellet total dry weight exhibited significant positive autocorrelation (Moran's I =

$2420.111, \mathrm{p}<0.001$, Fig. 2). We used generalized least squares (GLS) models to account for

243 autocorrelation in model residuals (Selmi and Boulinier 2001). Different models of spatial 
244 structure (assuming spherical, exponential and Gaussian structures) were fitted and the best

245 fitting model (exponential in all the cases) was defined using the Akaike Information Criterion

246 (AIC; Selmi and Boulinier 2001, Diniz-Filho et al. 2003).

247 We log transformed the pellet total dry weight to meet the assumptions of constant

248 variance and normality of the residuals. To avoid collinearity issues, we only considered models

249 including explanatory variables that were not significantly correlated (all $\mathrm{p}>0.14)$. We did not

250 include interactions among explanatory variables because of the small sample size. For patches

251 with no burrows within a 20 m of diameter circle $(n=7)$, the variable "distance to nearest burrow"

252 was missing. Hence, we first tested the effect of this variable on a subsample and then reran the

253 models without it to avoid artificially reducing sample size when testing the other explanatory

254 variables. We proceeded in the same way for "distance to nearest side" ( $\mathrm{n}=2$ patches with no

255 visible side) and for "mean distance to sides" (same 2 patches). We selected the final model by

256 fitting the complete model and removing each term successively. The significance of each term

257 was determined by assessing the change in deviance (i.e., Likelihood Ratio Test - LRT) against a

$258 \mathrm{chi}^{2}$ distribution with the appropriate degrees of freedom. For nonsignificant variables considered

259 in several models, we present the maximum LR value, the corresponding minimum p-value and

260 the estimates. Estimates were all computed on standardized variables $($ mean $=0$, S.D. $=1)$ to

261 allow comparisons of effect sizes not dependent on measurement scale (Gelman and Hill 2007).

262 Analyses were performed in R 3.1.2 (R Core Team 2014) using the packages ape for spatial

263 analyses and nlme for developing the models. The French Polar Research Institute approved this

264 program (number 279).

265

266 Results 
The unobstructed area within a $25 \mathrm{~m}$ radius circle around the patch positively affected the

269

270

271

272

273

274

275

276

277

278

279

280

281

282

283

284

285

286

287

288

289

pellet total dry weight $(\mathrm{df}=1, \mathrm{LR}=9.264, \mathrm{p}=0.002$; estimates: intercept $=1.41 \pm 0.20$ S.E., slope $=0.51 \pm 0.16$ S.E.; Fig. 3). When the unobstructed area increased from 500 to $1000 \mathrm{~m}^{2}$, the predicted pellet total dry weight increased from 3.38 to $7.02 \mathrm{~g}$.

The mean distance to visible mound sides and, to a lesser extent, the distance to the nearest visible side were also positively related to the pellet total dry weight (mean distance to mound sides: $\mathrm{df}=1, \mathrm{LR}=4.721, \mathrm{p}=0.030$; estimates: intercept $=1.40 \pm 0.23$ S.E., slope $=0.39$ \pm 0.17 S.E.; distance to the nearest side: $\mathrm{df}=1, \mathrm{LR}=3.776, \mathrm{p}=0.052$; estimates: intercept $=$ $1.39 \pm 0.24$ S.E., slope $=0.35 \pm 0.18$ S.E.). Finally, the total number of visible sides within a 25 $m$ circle negatively affected the pellet total dry weight $(d f=1, L R=5.032, p=0.025$; estimates: intercept $=1.42 \pm 0.27$ S.E., slope $=-0.37 \pm 0.16$ S.E.).

The other explanatory variables, including the distance to the nearest mound, were not retained in the final models and had smaller effect sizes as measured by standardized regression coefficients (distance to the nearest mound: $d f=1, L R=2.156, p=0.142$; estimates: intercept $=$ $1.44 \pm 0.27$ S.E., slope $=0.25 \pm 0.17$ S.E.; total number of old burrows: $\mathrm{df}=1, \mathrm{LR}=0.247, \mathrm{p}=$ 0.619; estimates: intercept $=1.40 \pm 0.19$ S.E., slope $=-0.08 \pm 0.14$ S.E.; distance to the nearest old burrow: $\mathrm{df}=1, \mathrm{LR}=1.847, \mathrm{p}=0.174$; estimates: intercept $=1.27 \pm 0.51$ S.E., slope $=-0.27$ \pm 0.18 S.E. ; isolation: $\mathrm{df}=1, \mathrm{LR}=1.014, \mathrm{p}=0.314$; estimates: intercept $=1.41 \pm 0.31$ S.E., slope $=-0.15 \pm 0.15$ S.E.).

The hypothesis that the proximity to active burrows partly explains the spatial pattern we report (Fig. 2) might be relevant as active burrows were localized at about 100 meters northwest from the patches, i.e., on the side of the highest values in pellet total dry weight. 
the coefficient of determination between the observed and the fitted values), in line with our field observations of cat hunting behaviors (Fig. 4).

293

294

\section{Discussion}

We took advantage of a study area with patches composed of a single preferred plant

297

298

299

300

301

302

303

304

305

306

307

308

309

310

311

312

species, displaying cover objects of a single type (i.e., a single intrinsic obstructive/protective ratio) and where predation risk arose from only one species, feral cats. In this context, our results strongly suggest that cats shape fine-scale habitat use by rabbits. Fewer pellets were present in patches with smaller unobstructed areas, i.e., with smaller visible area from the center of a $25 \mathrm{~m}$ radius circle around the patch, and thus closer potential danger, and with greater proximity of physical barrier, and thus reduced escape possibilities.

The consideration of the area to cover helped to understand the role of cover in fine-scale habitat use by rabbits. With classical approaches, i.e., with the distance to the "nearest cover" (i.e., nearest mound or nearest visible side and thus shortest time to initiate an escape and shortest distance before reaching a barrier to escape/an exit) as an explanatory variable, the conclusion of an absence of (or weak) differences between the patches in the amount of pellets according to the proximity of cover would have emerged (i.e., no or weak effect of nearest mound or nearest visible side distance in our analyses, respectively). Yet, our results strongly suggest that proximity to cover shapes habitat use by rabbits in this habitat. Our method requires no particular expertise - we provide an R-script to calculate the required areas (see Supplemental information file 2). Although a rangefinder with angle displays 
313 may be expensive, this can be replaced by a basic model without angle displays (or even by a

314 tape measure) and a compass. A limitation of our method is the subjectivity in classifying a

315 portion of the cover object as rectilinear or not. However, this subjectivity is more pronounced

316 when the cover is far, i.e., when the rabbit also faces visual limitations.

317 Beside the role of predation risk as a driver of the pattern we report, we cannot rule out

318 the influence of nonexclusive additional pressures. Reduced visibility may also lead to decreased

319 opportunity to monitor conspecifics while foraging. This may again indirectly relate to predation

320 risk because of the concomitant loss of information about conspecifics' vigilance/escape

321 behavior and because of the decreased "confusion effect" (Beauchamp 2014), as other prey

322 individuals are also less visible to an attacking predator. However, reduced visibility is further

323 expected to decrease foraging and social opportunities (e.g., localization of high quality patches,

324 scrounging, gathering information about potential mates/competitors) (Beauchamp 2014 for a

325 review, Monclús and Rödel 2008 for rabbits). Smaller unobstructed area is also expected to

326 mechanistically lead to lower overall surface of edible plants (i.e., more mounds and thus less

327 accessible plants per unit area) and thus to increased foraging effort/gram of ingested grass/unit

328 time, i.e., to possible decreased attractiveness. Finally, we cannot rule out the possibility that

329 patches with small unobstructed areas are more difficult to find for rabbits.

330 Pellet weight suggests that rabbits spend more time in patches with larger unobstructed

331 areas. However, the similar height of grass suggests that different patches are foraged at similar

332 rates which would be expected to take similar amounts of time. Several hypotheses may explain

333 this apparent contradiction. First, because we did not measure grass height, we cannot rule out

334 the possibility that patches with larger unobstructed areas had slightly shorter grasses as a result

335 of more time spent foraging. Second, although we believe meteorological and edaphic conditions 
336 are very similar over our restricted study area, we cannot rule out the possibility of fine-scale

337 variations. This could lead to higher Poa annua productivity in some patches, and thus to

338 increased foraging time despite similar height of grass. For instance, patches with larger

339 unobstructed areas may benefit from increased sunlight. Finally, under the hypothesis of similar

340 foraging rates among patches, behavioral explanations may be proposed. Rabbits may increase

341 grazing rate (through bite rate/size) and thus decrease exposure time in patches with smaller

342 unobstructed areas. Additional activities such as grooming, resting or playing may also occur in

343 safer places (e.g., Cowlishaw 1997, Blumstein 1998), leading to an increase in the overall

344 amount of time spent.

346 Past and future studies

347 The use of an incomplete measure of the proximity to cover may explain the absence of

348 significant results in studies investigating behavioral adjustments of prey in relation to cover

349 (e.g., Pays et al. 2014) while other studies in the same population report such an effect (e.g.,

350 Périquet et al. 2012), depending on which particular cover object is considered. Investigating the

351 predictive power of our proxy in these prey-predators-habitats systems, including large-scale

352 cover objects such as forest edges, may be informative. Previous studies on habitat selection by

353 rabbits in relation to cover reported contrasting results, possibly reflecting the ambivalent

354 properties of cover and the large range of predators rabbits face in their worldwide distribution

355 (Courchamp et al. 2003). While the importance of the protective function of cover in rabbit

356 habitat selection has been shown by several studies (Villafuerte and Moreno 1997, Dellafiore et

357 al. 2014), leading to a greater use of patches farther away from cover when predation pressure is

358 lower (Banks et al. 1999), cover may also be avoided when it reduces the visual field (Moreno et 
359 al. 1996). Part of the variability among studies may further be explained by the use of the

360 distance to nearest cover, widespread in this species also (e.g., Moreno et al. 1996, Villafuerte

361 and Moreno 1997). Moreover, the obstructive/protective ratio of a given cover for a given

362 population may vary according to the period of the day (Moreno et al. 1996): rabbits have been

363 reported to preferentially feed closer to cover during the day (hiding from birds of prey) than at

364 night (avoiding stalking carnivorous mammals).

365 Cover presenting total visual and physical obstruction, as in our study, is probably

366 uncommon. Assessing the obstructive/protective ratio and thus the risk perception associated

367 with a cover may require consideration of its visual and physical properties (Schooley et al.

368 1996, Camp et al. 2012). Moreover, it is probably uncommon to have only a single type of cover

369 as in our study. The "area to cover" should thus be calculated separately for each cover type.

370 Cover objects can all be of the same nature (e.g., protective/obstructive properties $>1$ ) but differ

371 in the relative intensity of protective and obstructive properties, or can be of opposite nature

372 (e.g., some with protective/obstructive properties $>1$ and some others with a ratio $<1$ ). For

373 example, a feeding patch for a mountain ungulate in the vicinity of a cliff and of several shrubs

374 and rocks may be characterized by the overall area to the shrubs and rocks (with a risk increased

375 for patches with smaller areas) and by the overall area to the cliff (with a risk decreased for

376 patches with smaller areas) if the individuals face stalk-and-ambush predators unable to reach

377 their prey on a cliff.

378

379 Acknowledgments 
380 We thank Fabien Egal, Johan Chervaux and Adrien Tavernier for field assistance, Gaël

381 Grenouillet for help with spatial analyses, two anonymous referees and editor D. L. Kramer for 382 very helpful comments on earlier drafts. 


\section{$383 \quad$ Fig.1}

384 The different potential cases for the position of edges of cover objects in relation to a circle

385 around the patch and the corresponding formulas for the calculation of the area ("A" in (B))

386 between the center of the patch and the cover object or the circle. The unobstructed area is

387 calculated by summing all these areas (see also Supplemental information file 2). Cases

388 numbered (1) to (4) in (A) refer to the same numbers in (B). Dark grey areas in (B) refer to

389 subcases illustrated in (A); light grey is used for alternative subcases. Inequalities in (B) are not

390 strict: for limiting cases, the different corresponding formulas lead to the same results. All the

391 angles are counterclockwise. $R$ is the radius of the circle. $d, \alpha, \beta_{1}$ and $\beta_{2}$ are defined in (C). We

392 defined $r_{1} \leq r_{2}$. The only required field measurements are $r_{1}, r_{2}$ and $\theta$. 
(A) An overview of a theoretical patch with the four main cases for the position of edges of cover objects

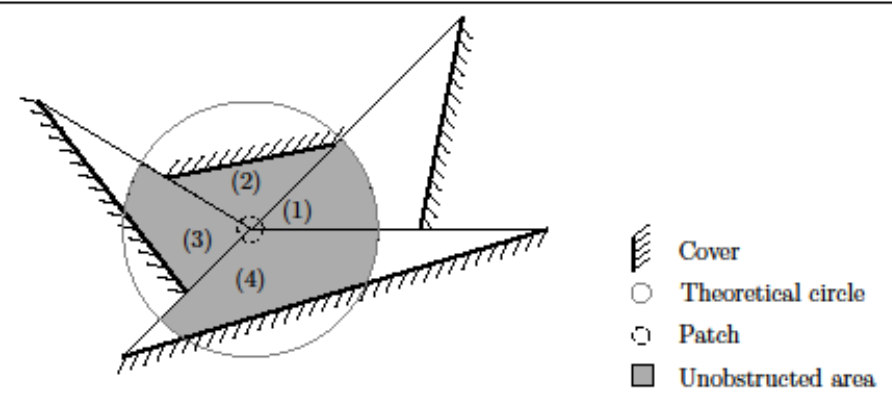

(B) The four main cases and the calculation of the surface for the associated subcases

(1) The whole edge of the cover object falls outside the theoretical circle

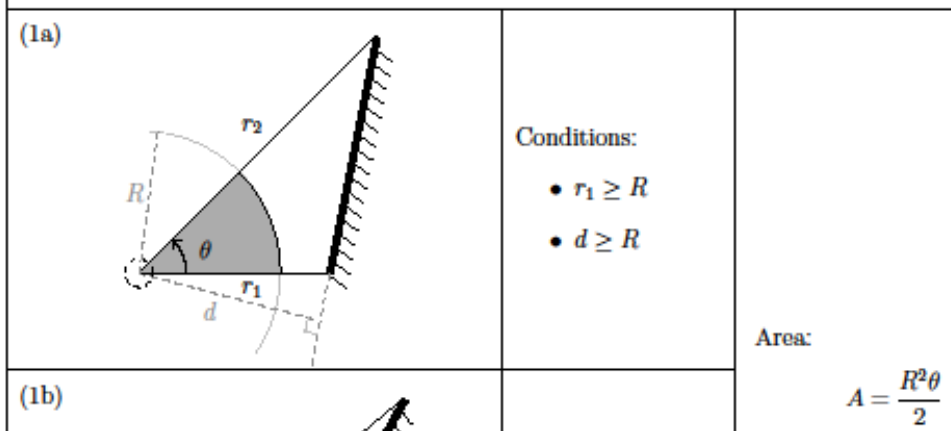

$(1 \mathrm{~b})$

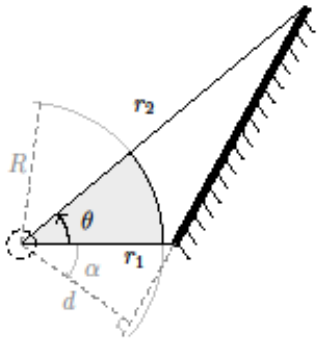

Conditions:

- $r_{1} \geq R$

- $d \leq R$

- $\alpha \leq 0$

(2) The whole edge of the cover object falls inside the theoretical circle

\begin{tabular}{l|l|l}
\hline & Conditions: \\
$\bullet r_{2} \leq R$ & Area:
\end{tabular}


Fig.1 (continued)

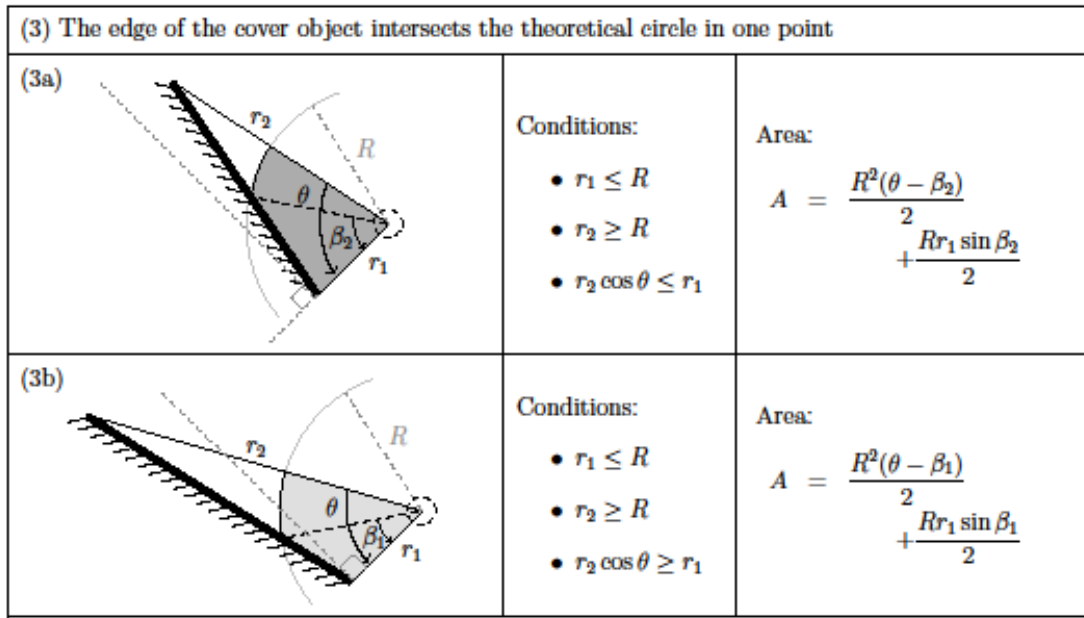

(4) The edge of the cover object intersects the theoretical circle in two points

\begin{tabular}{l|l|l} 
& Conditions: \\
& $\bullet r_{1} \geq R$ \\
& $\bullet d \leq R$ \\
& $\bullet \alpha \geq 0$ & $A=\frac{R^{2}\left(\theta-\beta_{2}+\beta_{1}\right)}{2}$ \\
&
\end{tabular}

\section{(C) Notations and formulas}

- Minimal distance (altitude length) between the center of the patch and the cover object:

$$
d=r_{1} r_{2} \frac{|\sin \theta|}{\sqrt{r_{1}^{2}+r_{2}^{2}-2 r_{1} r_{2} \cos \theta}}
$$

- Angle between the altitude and the border of the sector:

$$
\alpha=\arctan \frac{r_{1}-r_{2} \cos \theta}{r_{2} \sin \theta}
$$

- Angle of the intersection between the cover object and the theoretical circle:

$$
\begin{aligned}
& \beta_{1}=\arccos \frac{r_{1} r_{2}^{2} \sin ^{2} \theta+\sqrt{\left(R^{2}\left(r_{1}^{2}+r_{2}^{2}-2 r_{1} r_{2} \cos \theta\right)-r_{1}^{2} r_{2}^{2} \sin ^{2} \theta\right)\left(r_{2} \cos \theta-r_{1}\right)^{2}}}{R\left(r_{1}^{2}+r_{2}^{2}-2 r_{1} r_{2} \cos \theta\right)} \\
& \beta_{2}=\arccos \frac{r_{1} r_{2}^{2} \sin ^{2} \theta-\sqrt{\left(R^{2}\left(r_{1}^{2}+r_{2}^{2}-2 r_{1} r_{2} \cos \theta\right)-r_{1}^{2} r_{2}^{2} \sin ^{2} \theta\right)\left(r_{2} \cos \theta-r_{1}\right)^{2}}}{R\left(r_{1}^{2}+r_{2}^{2}-2 r_{1} r_{2} \cos \theta\right)}
\end{aligned}
$$


$398 \quad$ Fig. 2

399 Pellet total dry weight (indexed by grey levels shown on the right y-axis) measured in the studied

400 patches (dots) according to their spatial position (left $\mathrm{y}$-axis and $\mathrm{x}$-axis).

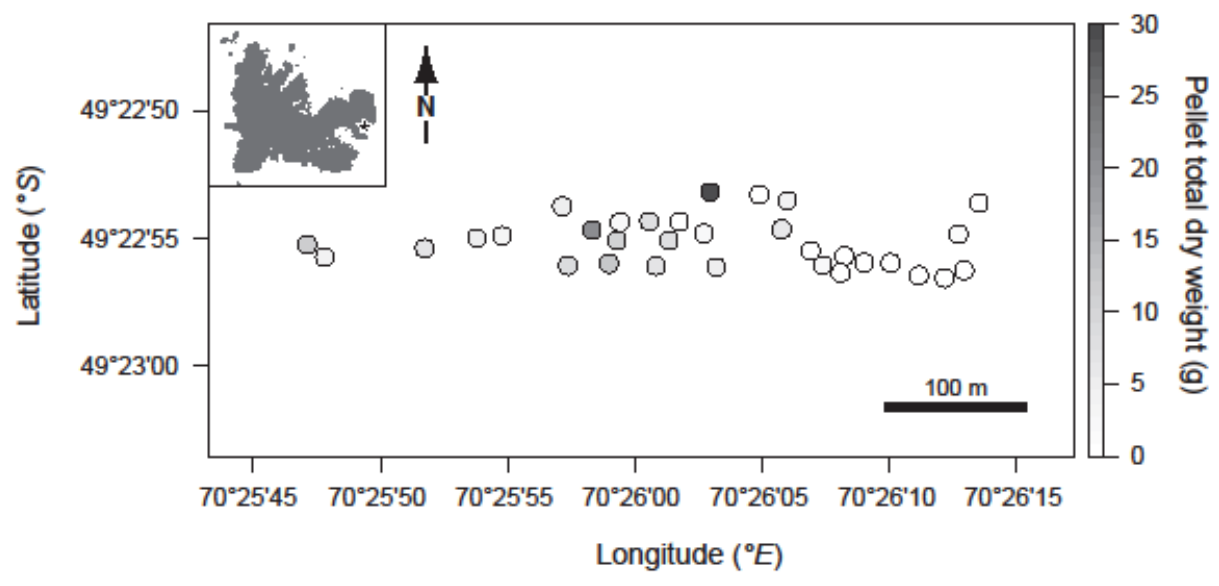


403 Fig. 3

404 The positive relationship between the $\log ($ pellet total dry weight $(g)+1)$ and the unobstructed

405 area $\left(\mathrm{m}^{2}\right)$ computed for a circle with a $25 \mathrm{~m}$ radius.

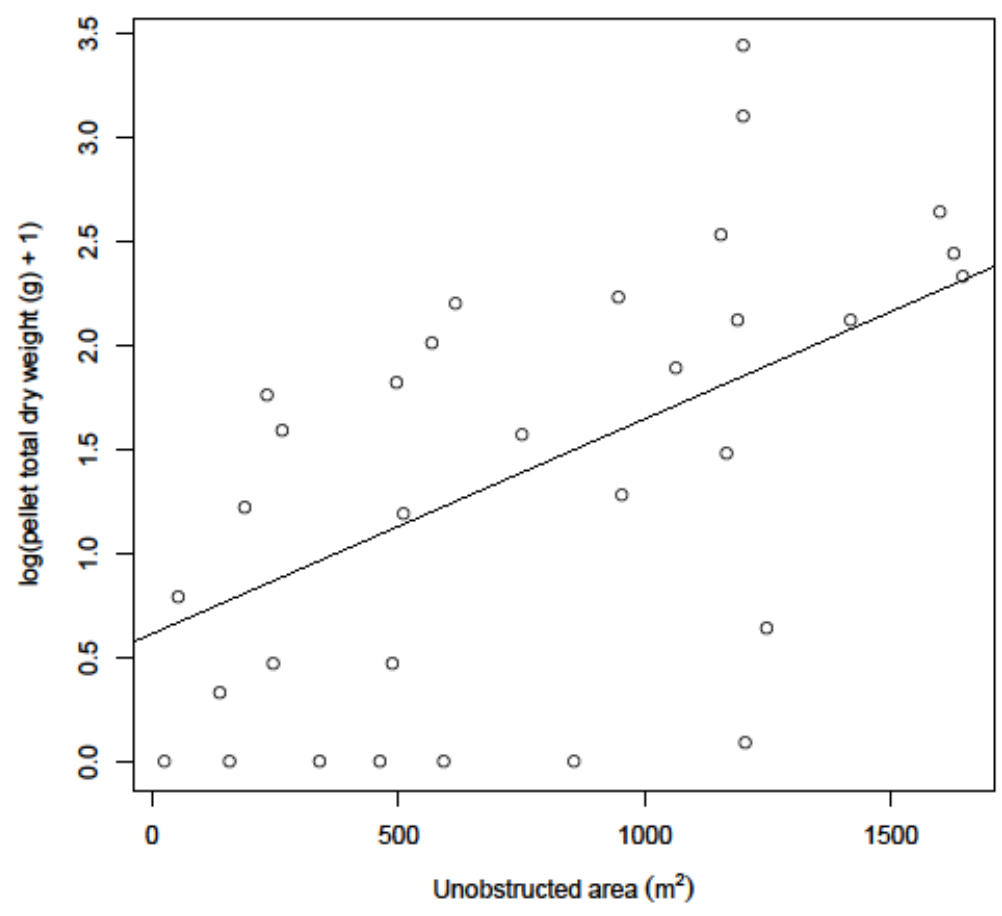

406 
408 Fig. 4

409 Squared coefficients of determination between the observed and the fitted values for models built

410 with values of unobstructed areas calculated for circles of radiuses ( $\mathrm{R}$ in Fig. 1, in $\mathrm{m}$ ) ranging

411 from 1 to $150 \mathrm{~m}$ and plotted on $\mathrm{x}$ axis. The biologically relevant distance ranges between 19 and

$41232 \mathrm{~m}$, in line with our field observations of cat hunting bouts.

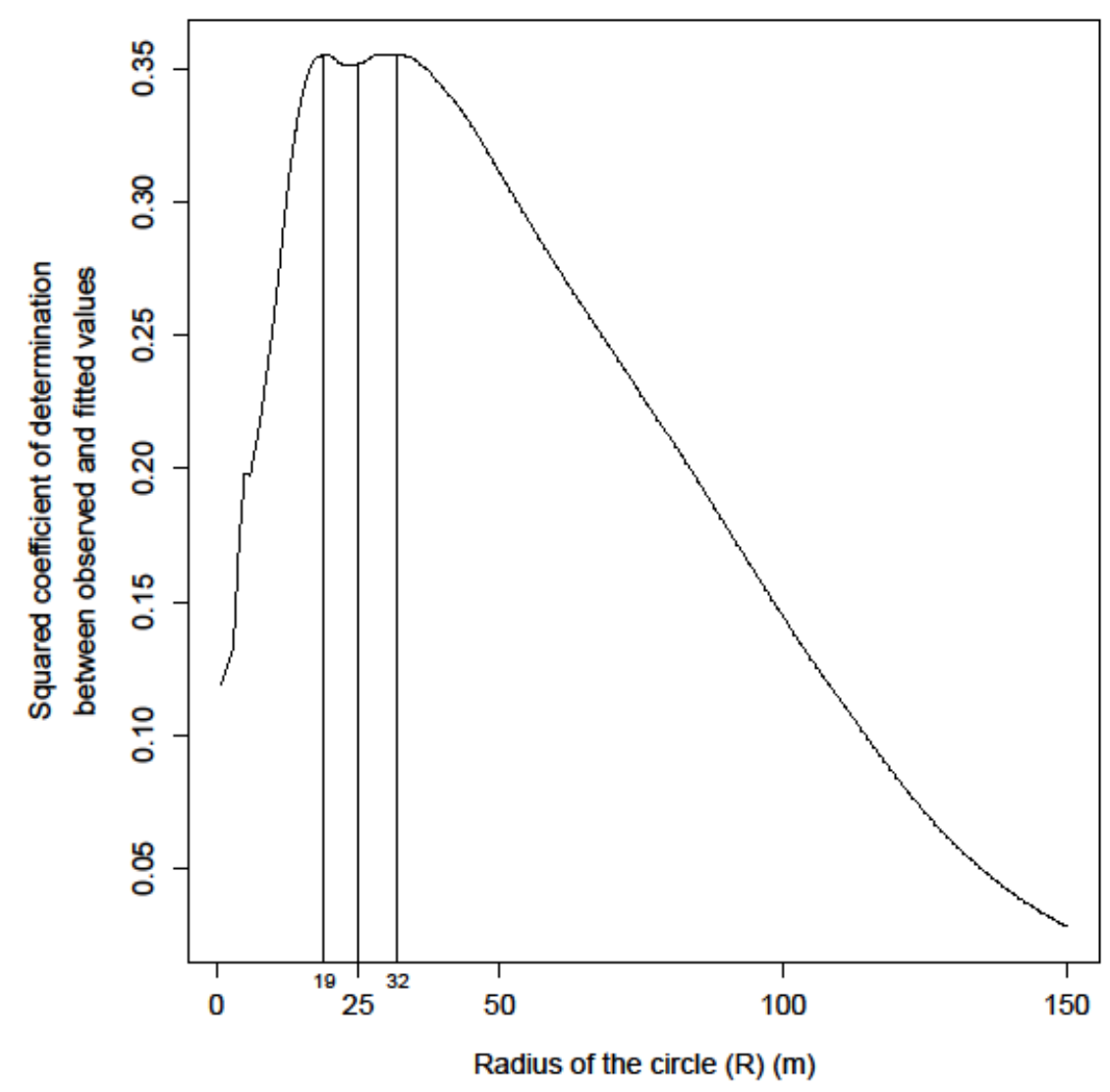


415

416

417

418

419

420

421

422

423

424

425

426

427

428

429

430

431

432

433

434

435

\section{References}

Bakker ES, Reiffers RC, Olff H, Gleichman JM. 2005. Experimental manipulation of predation risk and food quality: effect on grazing behaviour in a central - place foraging herbivore. Oecologia 146:157-167.

Ballinger A, Morgan, DG. 2002. Validating two methods for monitoring population size of the European rabbit (Oryctolagus cuniculus). Wildl. Res. 29:431-437.

Banks PB, Hume ID, Crowe O. 1999. Behavioural, morphological and dietary response of rabbits to predation risk from foxes. Oikos 85:247-256.

Beauchamp, G. 2014. Social Predation. Elsevier.

Blumstein DT. 1998. Quantifying predation risk for refuging animals: a case study with golden marmots. Ethology 104:501-516.

Bowyer RT, Van Ballenberghe V, Kie JG, Maier JAK. 1999. Birth-site selection by Alaskan moose: maternal strategies for coping with a risky environment. J. Mammal. 80:10701083.

Boussès P, Arthur CP, Chapuis JL. 1988. Rôle du facteur trophique sur la biologie des populations de lapins (Oryctolagus cuniculus L.) des Iles Kerguelen. Terre Vie - Rev. Ecol. A 43:329-343.

Burger J, Safina C, Gochfeld M. 2000. Factors affecting vigilance in springbok: importance of vegetative cover, location in herd, and herd size. Acta Ethol. 2:97-104.

Cabrera-Rodriguez F. 2006. Microhabitat selection of the European rabbit on La Palma, Canary Islands, Spain. Acta Theriol. 51:435-442. 
436 Camp M J, Rachlow JL, Woods BA, Johnson TR, Shipley LA. 2012. When to run and when to

437

438

439

440

441

442

443

444

445

446

447

448

449

450

451

452

453

454

455

456

457

hide: the influence of concealment, visibility, and proximity to refugia on perceptions of risk. Ethology 118:1010-1017.

Caro T. 2005. Antipredator defenses in birds and mammals. University of Chicago Press.

Chapuis JL, Boussès P, Barnaud G. 1994. Alien mammals, impact and management in the French subantarctic islands. Biol. Conserv. 67:97-104.

Courchamp F, Chapuis JL, Pascal M. 2003. Mammal invaders on islands: impact, control and control impact. Biol. Rev. 78:347-383.

Cowlishaw G. 1997. Refuge use and predation risk in a desert baboon population. Anim. Behav. 54:241-253.

Dellafiore CM, Rouco C, Muñóz Vallés S, Gallego Fernández JB. 2014. Seasonal habitat use by the European rabbit (Oryctolagus cuniculus) in a coastal dune system in SW Spain. Anim. Biodiv. Conserv. 37:233-242.

Diniz-Filho JAF, Bini LM, Hawkins BA. 2003. Spatial autocorrelation and red herrings in geographical ecology. Global Ecol. Biogeogr. 12:53-64.

Gelman A, Hill J. 2007. Data analysis using regression and multilevel/hierarchical models. Cambridge University Press.

Gómez-Serrano MA, López-López P. 2014. Nest site selection by Kentish Plover suggests a trade - off between nest - crypsis and predator detection strategies. PLoS ONE 9:e107121.

Götmark F, Hohlfält A. 1995. Bright male plumage and predation risk in passerine birds: are males easier to detect than females? Oikos 74:475-484. 
458 Jaksic FM, Soriguer RC. 1981. Predation upon the European rabbit (Oryctolagus cuniculus) in

459 Mediterranean habitats of Chile and Spain: a comparative analysis. J. Anim. Ecol.

460 50:269-281.

461 Lazarus J, Symonds M. 1992. Contrasting effects of protective and obstructive cover on avian

462

463

464

465

466

467

468

469

470

471

472

473

474

475

476

477

478

479 vigilance. Anim. Behav. 43:519-521.

Laundré JW, Hernández L, Ripple WJ. 2010. The landscape of fear: ecological implications of being afraid. Open Ecol. J. 3:1-7.

Lima SL. 1990. Protective cover and the use of space: different strategies in finches. Oikos 58: $151-158$.

Metcalfe NB. 1984. The effects of habitat on the vigilance of shorebirds: is visibility important? Anim. Behav. 32:981-985.

Monclús R, Rödel HG. 2008. Different forms of vigilance in response to the presence of predators and conspecifics in a group living mammal, the European Rabbit. Ethology 114:287-297.

Moreno S, Delibes M, Villafuerte R. 1996. Cover is safe during the day but dangerous at night: the use of vegetation by European wild rabbits. Can. J. Zool. 74:1656-1660.

Morgantini LE, Hudson RJ. 1985. Changes in diets of wapiti during a hunting season. J. Range Manage. 38:77-79.

Murray DL, Boutin S, O'Donoghue M, Nams VO. 1995. Hunting behaviour of a sympatric felid and canid in relation to vegetative cover. Anim. Behav. 50:1203-1210.

Mysterud A, Østbye E. 1999. Cover as a habitat element for temperate ungulates: effects on habitat selection and demography. Wildlife Soc. B 27:385-394. 
480 Newberry RC, Shackleton DM. 1997. Use of visual cover by domestic fowl: a Venetian blind

481

482

483

484

485

486

487

488

489

490

491

492

493

494

495

496

497

498

499

500

501

502

effect? Anim. Behav. 54:387-395.

Palomares F, Delibes M. 1997. Predation upon European rabbits and their use of open and closed patches in Mediterranean habitats. Oikos 80:407-410.

Palomares F. 2001. Comparison of 3 methods to estimate rabbit abundance in a Mediterranean environment. Wildlife Soc. B 29:578-585.

Périquet S, Todd-Jones L, Valeix M, Stapelkamp B, Elliot N, Wijers M, Pays O, Fortin D, Madzikanda H, Fritz H, Macdonald DW, Loveridge AJ. 2012. Influence of immediate predation risk by lions on the vigilance of prey of different body size. Behav. Ecol. 23:970-976.

Pays O, Blanchard P, Valeix M, Chamaillé-Jammes S, Duncan P, Périquet S, Lombard M, Ncube G, Tarakini T, Makuwe E, Fritz H. 2012. Detecting predators and locating competitors while foraging: an experimental study of a medium - sized herbivore in an African savanna. Oecologia 169:419-430.

Pays O, Ekori A, Fritz H. 2014. On the advantages of mixed-species groups: impalas adjust their vigilance when associated with larger prey herbivores. Ethology 120:1207-1216.

Pontier D, Say L, Debias F, Bried J, Thioulouse J, Micol T, Natoli E. 2002. The diet of feral cats (Felis catus L.) at five sites on the Grande Terre, Kerguelen archipelago. Polar Biol. 25:833-837.

R Core Team. 2014. R: A language and environment for statistical computing. $\mathrm{R}$ foundation for Statistical Computing, Vienna, Austria. URL http:/www.R-project.org/

Schooley RL, Sharpe PB, Van Horne B. 1996. Can shrub cover increase predation risk for a desert rodent? Can. J. Zool. 74:157-163. 
503 Selmi S, Boulinier T. 2001. Ecological biogeography of Southern Ocean Islands: the importance 504 of considering spatial issues. Am. Nat. 158:426-437.

505 Stratmann A, Taborsky B. 2014. Antipredator defences of young are independently determined 506 by genetic inheritance, maternal effects and own early experience in mouthbrooding $507 \quad$ cichlids. Funct. Ecol. 28:944-953.

508 Tchabovsky AV, Krasnov BR, Khokhlova IS, Shenbrot GI. 2001. The effect of vegetation cover 509 on vigilance and foraging tactics in the fat sand rat Psammomys obesus. J. Ethol. 19:105$510 \quad 113$.

511 Villafuerte R, Moreno S. 1997. Predation risk, cover type, and group size in European rabbits in 512 Doñana (SW Spain). Acta Theriol. 42:225-230. 